\title{
Transformations et inerties du bénévolat associatif sur la période 1982-2002
}

\author{
Denis Bernardeau-Moreau ${ }^{2}$ \\ Matthieu HÉLY ${ }^{3}$
}

La figure contemporaine du bénévolat associatif tend à devenir plus complexe. Le faux-semblant d'une crise annoncée du bénévolat masque en réalité une profonde mutation de l'engagement associatif. L'analyse longitudinale du profil socioculturel et socioprofessionnel des adhérents dans deux enquêtes INSEE de 1982 et 2002 révèle que l'activité associative mobilise désormais autant les qualités et l'expérience professionnelle des adhérents que leurs convictions.

Les discours des acteurs du monde associatif évoquent souvent le thème d'un "déclin du bénévolat» et stigmatisent l'indivualisme contemporain comme la cause principale de leurs difficultés. Les données statistiques qui permettent, malgré leurs limites, de mesurer les évolutions de la participation associative confirment-elles ce sentiment?

Le monde associatif étant particulièrement complexe, il est essentiel de distinguer différentes catégories de «participants »: les bénévoles, les adhérents et les salariés. Les bénévoles associatifs seraient aujourd'hui environ 10 millions. En comparaison avec des enquêtes plus anciennes ${ }^{4}$, on constate que leur nombre a augmenté (en 1990, ils étaient 7,9 millions, en 1993, 9,1 millions et en 1996, 10,4 millions soit 23,4\% de la population de plus de 18 ans). Parmi ces bénévoles, il convient de distinguer les dirigeants (i.e. qui détiennent des responsabilités dans l'association en tant qu'administrateur ou membre d'un bureau exécutif)

1. (Analyse secondaire des enquêtes INSEE Contacts 1982-83 et EPCV 2002.)

2. Maître de conférences, Université de Marne-la-Vallée - Analyse comparée des pouvoirs.

3. IDHE, Université Paris $\mathrm{X}$ - Maison Max-Weber, Nanterre.

4. Notamment Archambault E., Bon C., Le Vaillant M. et Boumendil J., «Les dons et le bénévolat en France », enquête LES-ISL Fondation de France, 1991-1994-1997, Paris. 
des participants réguliers et occasionnels ${ }^{5}$. Pour autant, il n'est pas possible de définir un profil-type de bénévole. Cela dépend des missions remplies par les bénévoles, des secteurs investis, de leurs niveaux de participation, de leurs niveaux de responsabilité. D’après les enquêtes, le bénévole associatif en France et en Europe est plutôt un homme âgé de 35 à 54 ans, issu d'un milieu plutôt aisé et cultivé, possédant un capital scolaire élevé et exerçant ou ayant exercé une activité professionnelle en milieu moyennement urbain. Ces divers constats soulignent que le monde associatif est bien loin d'être confronté à une « crise du bénévolat » et que ce registre de dramatisation, fréquemment invoqué, masque en réalité une profonde transformation des formes et des modalités d'exercice de la pratique bénévole ${ }^{6}$. Concernant les adhérents stricto sensu, l' INSEE ${ }^{7}$ nous rapporte que si huit Français sur dix se disent concernés par la vie associative, ils sont $39 \%$ en 1997 (soit 20 millions de Français de plus de 14 ans) à être adhérents dans une association. $A$ priori, cela fait autant qu'en 1983. L'adhésion concerne toutes les tranches d'âge mais semble avoir davantage augmenté dans les secteurs sportif, culturel et humanitaire qu'ailleurs. Si, en effet, entre 1983 et 1996, le taux d'adhésion dans les clubs sportifs est passé de 15 à $18 \%$ et dans le secteur humanitaire de 2 à $4 \%$, dans les syndicats et groupements professionnels il a chuté pour la même période de 14 à $8 \%$ et dans les associations de parents d'élèves de 12 à $8 \%$. Pour les auteurs de cette enquête, il faut voir dans ces évolutions conjointes les signes d'un engagement recherchant davantage l'accomplissement individuel qu'un idéal collectif. Pour ce qui est de l'évolution du nombre des associations, les statistiques sont moins fiables tant la part des associations en sommeil mais non dissoutes semble importante. Seules les créations d'associations recueillies par les préfectures sont des sources fiables : une étude publiée en 1999 par le $\mathrm{CREDOC}^{8}$ relève que si, en 1975, le nombre d'associations créées chaque année était de 20 000,

5. Hatchuel G., Loisel J.-P., «L'adhésion aux associations reste à un niveau élevé. Plus de seniors, moins de militants », Consommation et Modes de vie, CREDOC, ${ }^{\circ} 123$, janvier 1998. Les auteurs identifient différents degrés d'implication dans les associations.

6. Nous rejoignons notamment ici les travaux du réseau thématique 35 à l'occasion $\mathrm{du} 2^{\mathrm{e}}$ congrès de l'Association française de sociologie. Voir également l'article de Maud Simonet «Penser le bénévolat comme travail pour repenser la sociologie du travail», Revue de l'IRES, 2004, nº 44, p. 141-155.

7. Crenner E., «Le milieu associatif de 1983 à 1996 », Division Conditions de vie des ménages, INSEE Première, n 542, septembre 1997.

8. Fourel C., Loisel J.-P., « Huit Français sur dix concernés par la vie associative », Consommation et Modes de vie, CREDOC, n 133, 20 février 1999. 
aujourd'hui, le nombre de créations annuelles s'élève à 60000 (notons que ce nombre n'était que de 1000 par an en 1920 et de 5000 par an en 1945). Aux côtés des bénévoles et des adhérents, les salariés constituent le troisième acteur majeur dans l'association. Selon l'enquête du $\mathrm{CNRS}^{9}$, le nombre d'associations employeurs ne dépasserait pas 145000 sur un total de 880000 associations recensées. On estime ainsi que ces associations emploieraient plus de 1,6 million de personnes en France. Depuis une vingtaine d'années, la tendance va vers une augmentation du nombre de salariés dans le champ associatif, marquant ainsi une des formes de professionnalisation les plus significatives (rapport parlementaire de Derosier, octobre 2000).

Nous nous proposons dans cet article de prendre au sérieux l'idée d'une «professionnalisation » du bénévolat associatif en tentant d'en repérer les linéaments dans les données des enquêtes statistiques menées par l'INSEE depuis vingt ans. Notre intention est de souligner que si la salarisation est un indicateur très fort de la professionnalisation, elle n'en est pas pour autant le seul. La professionnalisation caractérise l'ensemble du monde associatif et se situe bien au-delà du bénévolat, se traduisant par l'explosion de l'emploi de salariés employés par des « entreprises associatives ${ }^{10}$. Par professionnalisation, nous entendons un processus de rationalisation à la fois en termes de compétence dont le degré de spécialisation devient plus élevé et en termes de statut s'exprimant par une salarisation mais aussi par l'institutionnalisation de professions (ne parle-t-on pas aujourd'hui de « bénévole professionnel » ou de "professionnel bénévole » ${ }^{11}$ ). Dans le cas du bénévolat associatif, nous voulons montrer que la professionnalisation s'exprime notamment par la participation bénévole qui requiert de plus en plus de compétences du fait de l'exercice de responsabilités particulières (la vie associative demanderait ainsi davantage de compétences administratives, gestionnaires et juridiques).

9. Enquête auprès des associations 1999 coordonnée par Viviane Tchernonog; cf. Tchernonog V., «Le financement de la solidarité. Les ressources des associations: éclairage à partir des financements publics et des réseaux », Rapport de fin de contrat pour la MIRE (Mission recherche-expérimentation du ministère de l'Emploi et de la Solidarité), 2000.

10. M. Hély, «Les différentes formes d'entreprises associatives », Sociologies Pratiques, ${ }^{\circ}$ 9, 2004, p. 27-51.

11. D. Bernardeau, «La professionnalisation dans les organisations associatives. Le cas des dirigeants bénévoles de la Fédération française d'équitation », Gérer et comprendre, Annales des Mines, $\mathrm{n}^{\circ} 73,2003$, p. 55-74. 
Notre stratégie de recherche a donc consisté en une analyse longitudinale du profil socioculturel et socioprofessionnel des adhérents (différenciation selon le degré de participation) d'une ou plusieurs associations (tous champs confondus) à partir de deux enquêtes INSEE réalisées en 1982-83 (enquête Contacts et notamment la partie variable «Étude sur les contacts entre les personnes ») et en 2002 (notamment la partie variable "Vie associative» de l'enquête permanente conditions de vie des ménages $(\mathrm{EPCV}))^{12}$. Notre intention, à travers cette étude comparative, est de deux ordres. Elle est de déterminer l'ensemble des éléments susceptibles de caractériser les principales évolutions du profil des adhérents en vingt ans. Des indicateurs comme les PCS, l'âge, le niveau d'étude, l'expérience associative et amateur, le degré de responsabilité, le cumul des mandats sont étudiés et comparés sur les deux périodes. À travers cela, notre intention est aussi de cerner, notamment chez les « dirigeants bénévoles » (c'est-à-dire les individus déclarant exercer des responsabilités au sein d'une association), les principales caractéristiques du processus de professionnalisation dont les définitions multiples témoignent de la difficulté à définir précisément cette tendance lourde. In fine, l'analyse secondaire des enquêtes INSEE, à partir de données ponctuelles répétées de 1133 responsables associatifs interrogés entre 1982 et 1983 et 559 en 2002, devra nous permettre de vérifier un ensemble d'hypothèses relatives à l'adhésion, la professionnalisation, le niveau d'engagement et les logiques d'intérêts de ces acteurs associatifs.

Nous relevons essentiellement deux tendances fortes : une inertie des déterminants sociodémographiques de l'adhésion et de la participation associative d'une part, et une baisse des cumuls des adhésions accompagnée d'une élévation de la participation associative d'autre part.

\section{Une forte inertie des déterminants sociodémographiques de l'adhésion et de la participation associative}

Toutes les enquêtes quantitatives réalisées en France depuis 1982, c'est-à-dire consécutives aux deux articles fondateurs de François Héran sur le monde associatif ${ }^{13}$, n'ont fait que confirmer le poids déterminant

12. Voir la note méthodologique en fin d'article pour plus de précisions.

13. Voir Héran F., «Un monde sélectif : les associations », Économie et Statistique, $\mathrm{n}^{\circ}$ 208, mars 1988, p. 17-31; et « Au cœur du réseau associatif : les multi-adhérents », Économie et Statistique, n 208, mars 1988, p. 33-44. 
des variables sociodémographiques comme le sexe, l'âge, la profession exercée et le diplôme sur la probabilité de participer à la vie associative ${ }^{14}$. Les résultats obtenus à partir des sources étudiées confortent l'idée selon laquelle l'influence de ces variables reste identique. Concernant la liaison entre le sexe et la participation associative, il convient de souligner que, en dépit d'une augmentation de la proportion de femmes déclarant adhérer à une association en y exerçant des responsabilités, l'écart avec les hommes ne diffère pas de façon significative de ce qu'il était il y a vingt ans : 8,4 points en 1982 contre 7,7 en 2002 (ce qui n'est pas suffisant du point de vue des intervalles de confiance). Les bonnes intentions qui ponctuent les discours consensuels sur la parité restent donc encore aujourd'hui de simples discours.

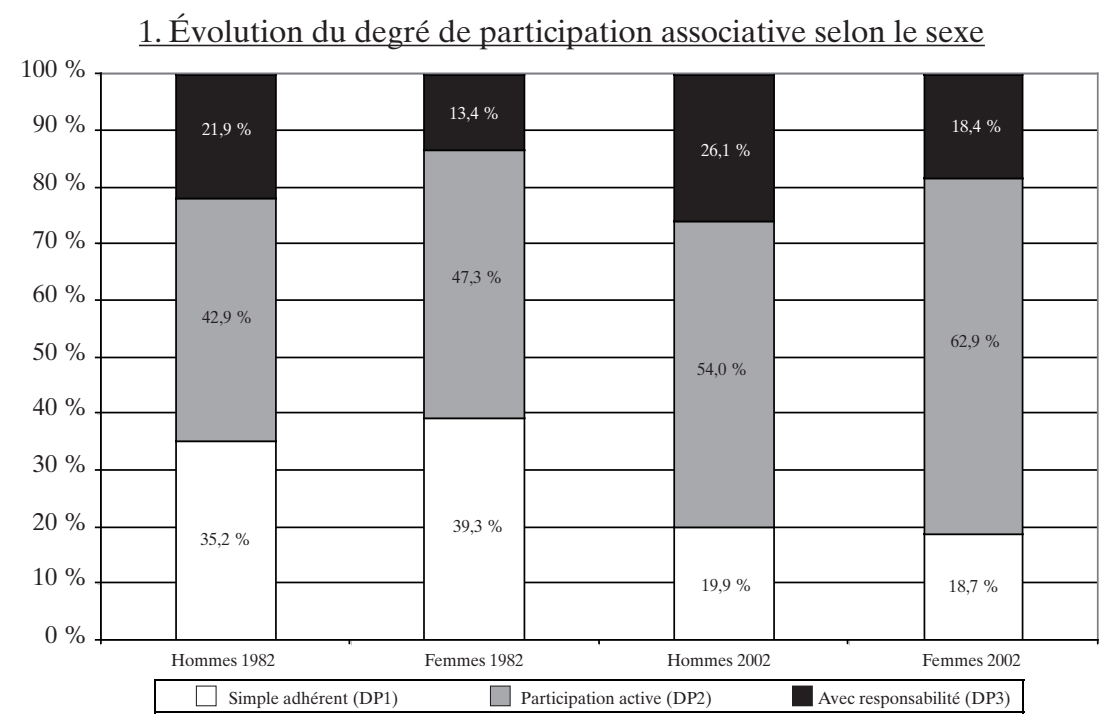

On constate peu de changement dans la probabilité d'adhérer à une association en fonction du groupe professionnel auquel on appartient sur la période 1982-2002. Les cadres et professions intellectuelles supérieures perdent une part de leur hégémonie sur le monde associatif, puisque leur adhésion subit une baisse de 10 points, mais cependant près des deux tiers d'entre eux déclarent adhérer à au moins une asso-

14. Voir Hatchuel G. et Loisel J.-P., « La vie associative : participer, mais pas militer », in Données sociales 1999-2000, INSEE, 2000, p. 359-365, et Crenner E., « Le milieu associatif de 1983 à 1996 : plus ouvert et tourné vers l'intérêt individuel », INSEE Première, $\mathrm{n}^{\circ} 542$, septembre 1997. 
ciation. Sans doute l'érosion de l'adhésion associative des cadres est-elle liée à la diffusion massive de cette catégorie dans la population active entre ces deux dates. Les artisans, commerçants et chefs d'entreprise connaissent aussi une décrue de leurs chances d'adhérer à une association. L'hétérogénéité de ce groupe et son affaiblissement sur la période considérée rendent l'interprétation délicate. Les agriculteurs ont sans doute compensé leur isolement et la décomposition progressive de leur cohésion interne par une adhésion associative accrue. Enfin, les ouvriers restent en marge de la participation à la vie collective et leur taux de participation se dégrade puisqu'un tiers d'entre eux seulement déclarent adhérer à une association.

2. Évolution de l'adhésion associative selon le groupe professionnel

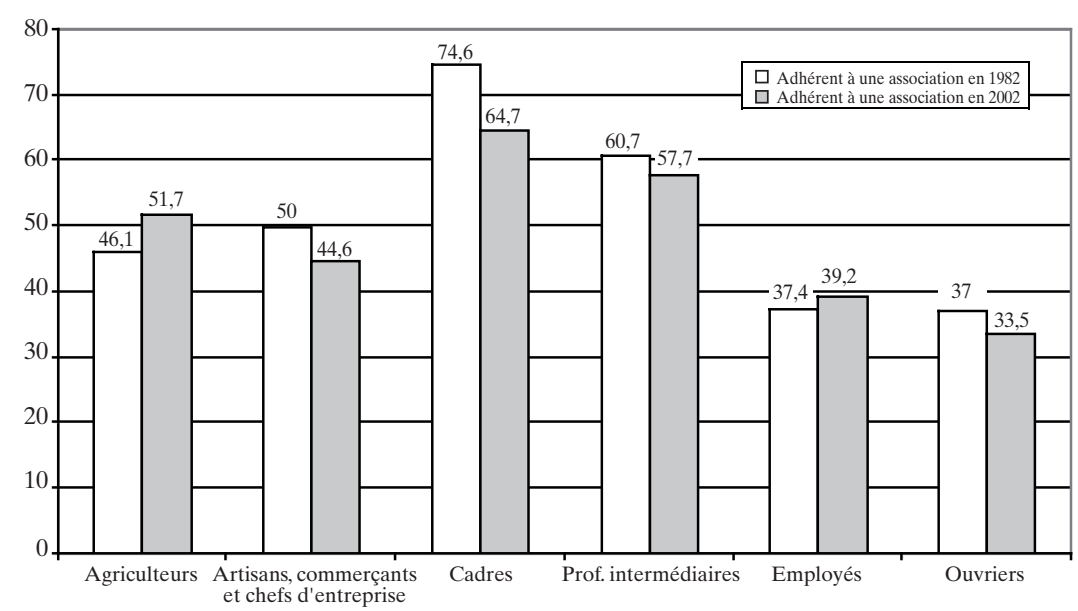

Le même constat peut être fait à propos des liens entre les groupes professionnels et le degré de la participation associative. On constate à nouveau un déclin sensible des chances de participer à une association, quelles que soient les modalités d'engagement (simple adhésion, participation active ou exercice de responsabilités), pour les ouvriers dont le poids dans la population active s'est certes atténué mais cependant pas dans les proportions observées ici (les ouvriers représentaient 31,4\% des individus enquêtés en 1982 dont la PCs était connue, contre 25,5\% en 2002). Globalement la ségrégation sociale reste très forte puisque les cadres et professions intermédiaires monopolisent les responsabilités : près d'un responsable associatif sur deux appartient à l'une ou l'autre de ces catégories bien qu'elles ne représentent qu'un tiers de la popu- 
lation active occupée. On note enfin un accroissement significatif de la participation des employés, en particulier en ce qui concerne l'accès aux responsabilités. On sait par ailleurs que les fonctionnaires sont fortement représentés dans cette catégorie, il est donc fort probable qu'ils tirent bénéfice ici d'horaires moins contraignants et d'une plus grande disponibilité que les salariés du secteur privé. C'est ce qu'avaient déjà relevé Lionel Prouteau et François-Charles Wolff dans leur étude sur l'effet du temps de travail sur la participation associative ${ }^{15}$.

3. Évolution du degré de participation associative selon le groupe professionnel

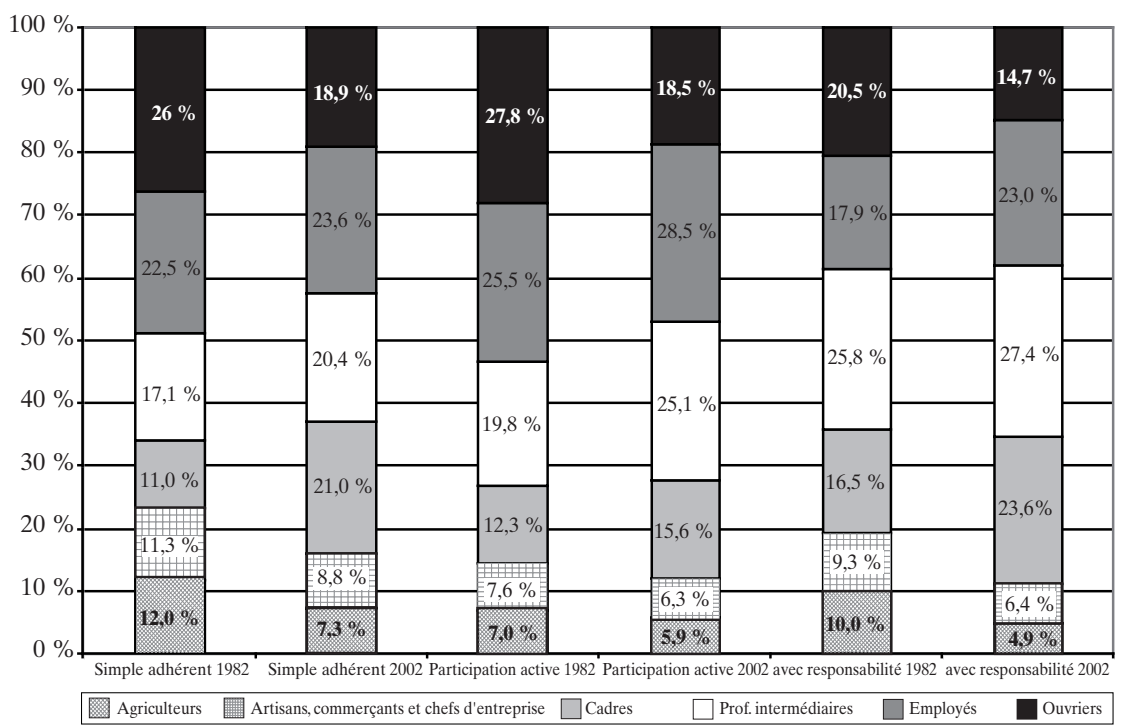

Parallèlement à la catégorie socioprofessionnelle, le niveau d'instruction s'avère également fortement discriminant. Les diplômés de l'enseignement supérieur sont ainsi ceux qui adhèrent et participent le plus aux activités associatives (cf. graphique 4). Ils représentent en 2002 plus du tiers des adhérents qui déclarent exercer des responsabilités dans le cadre de leur activité associative alors qu'ils n'étaient que $18 \%$ en 1982. À l'inverse la part des peu ou pas diplômés s'est effondrée à la fois sur la période et du point de vue du degré de participation (plus la participation est intense, plus la part des sans diplôme et des individus dotés du certificat d'études primaires comme le titre scolaire le plus élevé s'affaiblit).

15. Prouteau L. et Wolff F.-C., «La participation associative au regard des temps sociaux », in Économie et Statistique, no 352-353, p. 57-80. 


\section{4. Évolution de l'adhésion associative selon le diplôme}

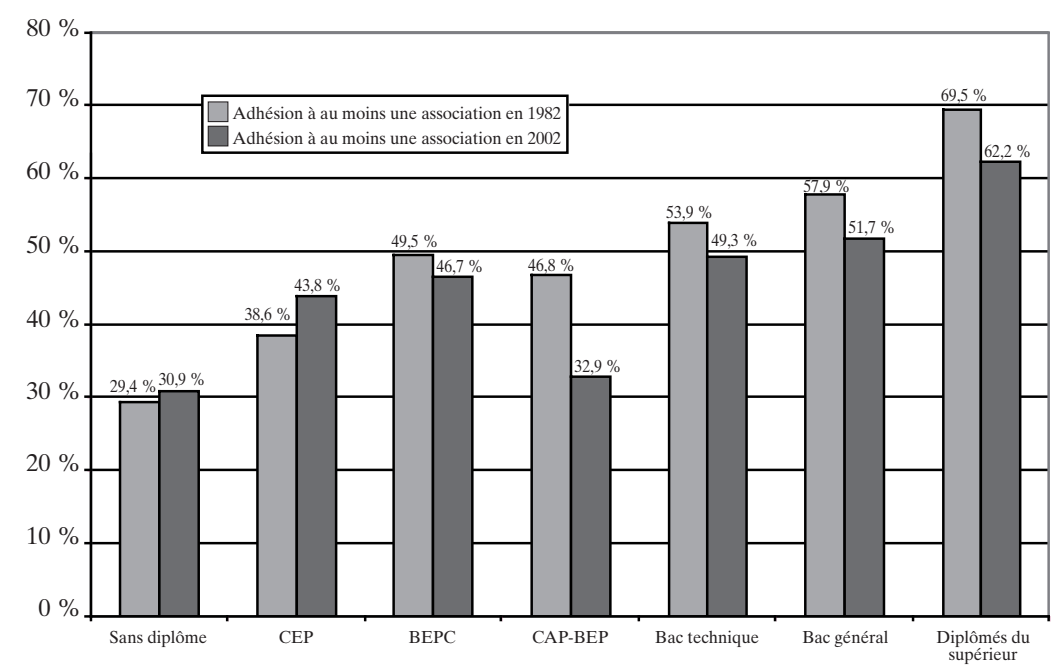

5. Évolution du degré de participation associative selon le diplôme

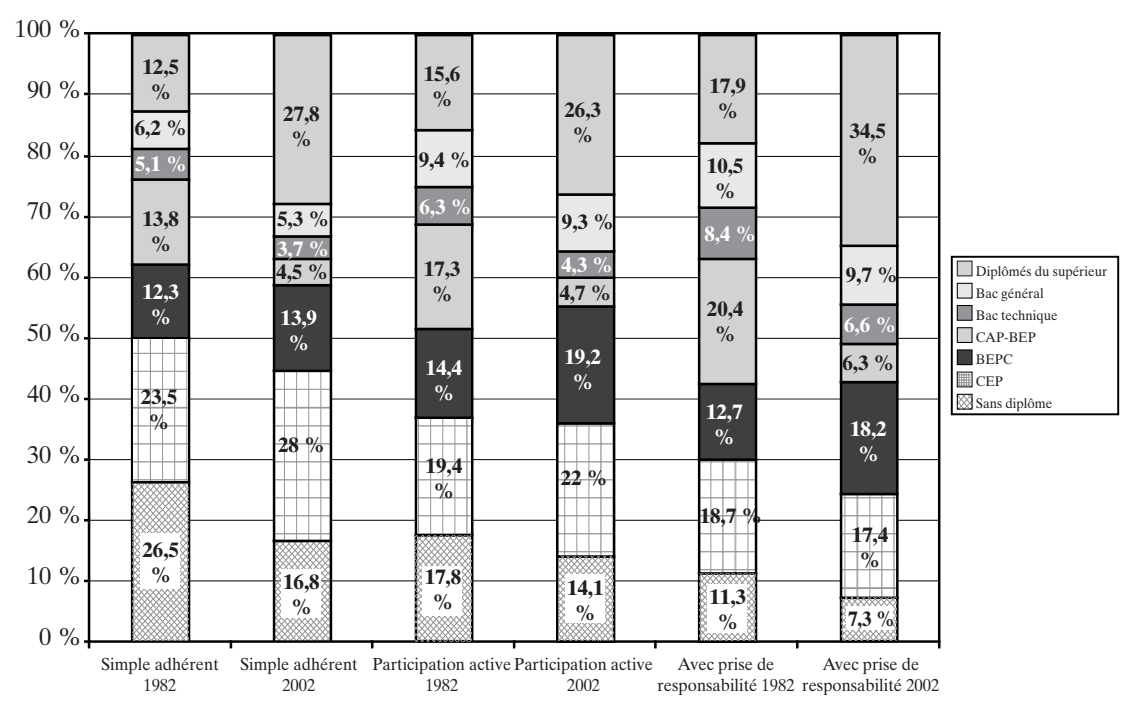


Mais il y a mieux. Pour contrôler les variations structurelles liées à la massification scolaire et à l'allongement des études, nous avons comparé la probabilité d'adhérer à une association en y exerçant des responsabilités avec les évolutions dans la structure des diplômes dans l'ensemble de la population enquêtée. Ainsi, la chance qu'un individu soit diplômé du supérieur plutôt que de ne pas l'être est deux fois plus importante en 2002 qu'en 1982 (cf. tableau des Odds ratio). Du point de vue des adhérents à au moins une association, on ne constate pas de différence significative. C'est-à-dire que la part des diplômés du supérieur parmi les adhérents à au moins une association n'a pas évolué plus vite que le reste de la population générale. En revanche, les «adhérents responsables» ont 2,42 fois plus de chances d'être diplômés du supérieur que de ne pas l'être. Ce qui signifie que cette catégorie s'est développée plus rapidement parmi les adhérents responsables qu'en population générale. La pratique associative, en particulier quand elle est effectuée dans le cadre de responsabilités institutionnelles, nécessite une capacité à incarner un collectif et à parler en son nom. Autrement dit, il est indispensable de se sentir légitime à représenter le groupe pour accéder au statut de « porte-parole ». Sur ce plan, l'acquisition de titres scolaires, en plus de faciliter l'expression orale et la maîtrise de la parole publique, place celui qui en dispose dans une situation légitime. À l'inverse, ceux qui en sont dépourvus s'autocensurent, faute de disposer d'une légitimité suffisante, et ce d'autant plus facilement qu'ils s'orientent plus fréquemment vers la « défection» que vers la «prise de parole », pour reprendre la distinction célèbre d'Albert Hirschmann ${ }^{16}$. Les discours incantatoires sur la « démocratie participative » se heurtent donc à des dispositions profondément ancrées dans les pratiques. Le déclin brutal du taux de participation des titulaires d'un CAP/BEP fait ici sans doute écho à l'affaiblissement du groupe ouvrier constaté dans le graphique précédent.

L'âge, enfin, contribue à structurer de façon significative la distribution des taux d'adhésion et de participation associative. Alors que ce taux culmine pour la tranche des 34 ans à moins de 44 ans en 1982, on constate que ce sont désormais les individus les plus âgés qui représentent les fréquences modales de la distribution. Effet d'âge ou de génération? Avec vingt ans de différence, nous retrouvons parmi les 54 ans et plus de 2002 les 34-54 ans de 1982. Seules des données longitudinales

16. Hirschmann A., Défection et prise de parole, Paris, Fayard, coll. «L'espace du politique », 1995. 


\begin{tabular}{|l|c|c|c|}
\cline { 2 - 4 } \multicolumn{1}{c|}{} & \multicolumn{3}{|c|}{ Odds ratio 2002/1982 } \\
\cline { 2 - 4 } \multicolumn{1}{c|}{} & $\begin{array}{c}\text { Adhésions à au moins } \\
\text { une association }\end{array}$ & $\begin{array}{c}\text { Adhésions avec prise } \\
\text { de responsabilité }\end{array}$ & Population générale \\
\hline Sans diplôme & 0,61 & 0,62 & 0,58 \\
\hline CEP & 1,09 & 0,91 & 0,98 \\
\hline BEPC & 1,53 & 1,54 & 1,72 \\
\hline CAP-BEP & 0,29 & 0,26 & 0,44 \\
\hline Bac technique & 1,16 & 0,78 & 1,34 \\
\hline Bac général & 1,04 & 0,91 & 1,23 \\
\hline Diplômés du supérieur & 1,84 & 2,42 & 2,11 \\
\hline
\end{tabular}

pourraient permettre de trancher sur ce point, mais il ne semble pas improbable que la cohorte ayant fait l'expérience des mouvements sociaux de mai 1968 soit restée particulièrement sensible à l'action collective menée dans le cadre associatif, et cela concerne autant l'adhésion que la participation avec prise de responsabilité.

6. Évolution de l'adhésion associative selon l'âge

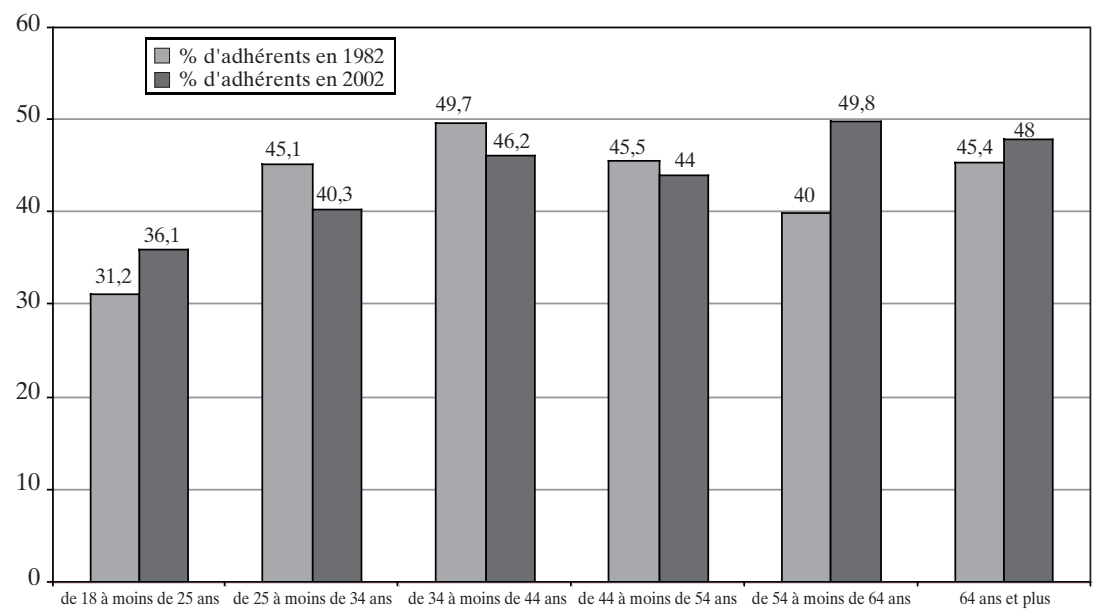

En conclusion de ce premier point, nous pouvons conclure à une assez forte inertie du profil sociodémographique de ceux qui adhèrent et/ou participent bénévolement. Il y a toujours plus d'hommes que de femmes, plus de cadres et de professions libérales que d'ouvriers, de plus en plus de gens diplômés du supérieur et un accroissement significatif des retraités. 
7. Évolution du degré de participation associative selon l'âge

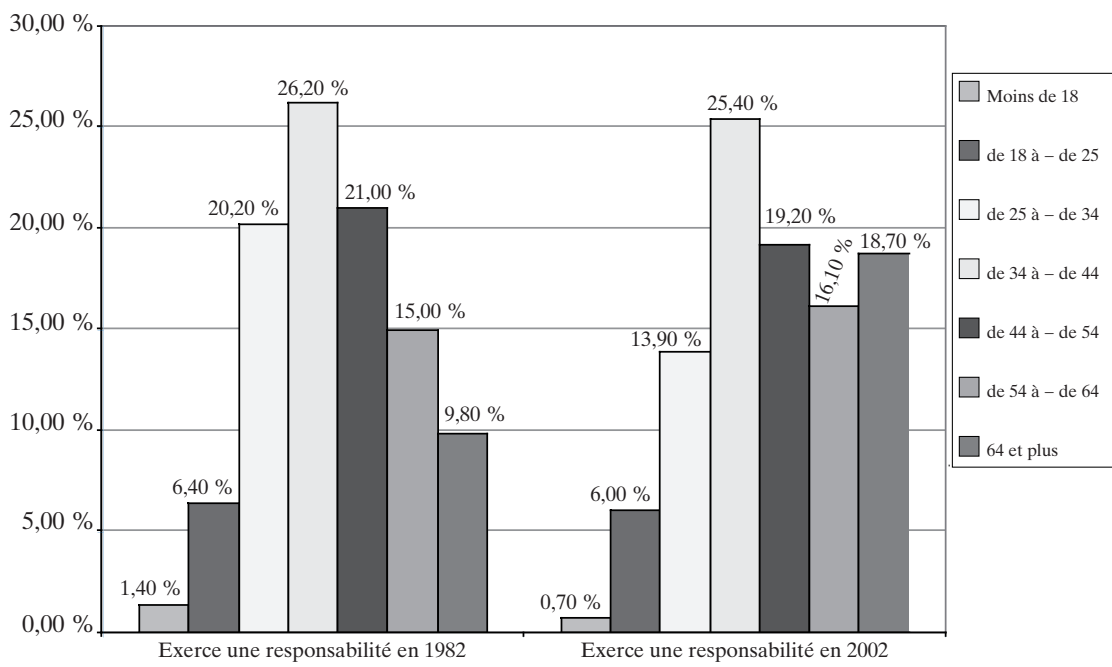

\section{Une participation associative moins dispersée mais plus dense}

Du point de vue du nombre d'associations auxquelles une personne déclare adhérer, il est intéressant de souligner une tendance générale à un moindre cumul des engagements (sauf, notons-le, pour les 64 ans et plus où, au contraire, le cumul augmente). Cette dispersion plus faible s'accomplit néanmoins dans le plus pur respect de la hiérarchie sociale puisque les « classes moyennes » (cadres et professions intermédiaires) restent dominantes du point de vue de la multi-adhésion dont François Héran avait déjà pointé le caractère discriminant.

En revanche, si entre 1982 et 2002 il y a moins de cumul d'adhésion, on observe une élévation globale du degré de participation (DP 2 et DP 3). Parmi les 6142 adhérents interrogés en 1982, on en compte 44,7\% déclarant participer activement dans leur association (DP 2) et 18,4\% déclarant prendre des responsabilités (DP 3). Il est intéressant de noter qu'en 2002 les adhérents déclarant participer activement ou déclarant prendre des responsabilités sont beaucoup plus nombreux (respectivement 60,1 et $21,3 \%$ ). En outre, le calcul des intervalles de confiance confirme que la différence entre 1982 et 2002 du point de vue de la proportion d'adhérents dont la participation est assortie d'une prise de 
$\underline{\text { 8. Nombre moyen d'adhésions par catégorie socioprofessionnelle }}$ parmi les adhérents d'au moins une association

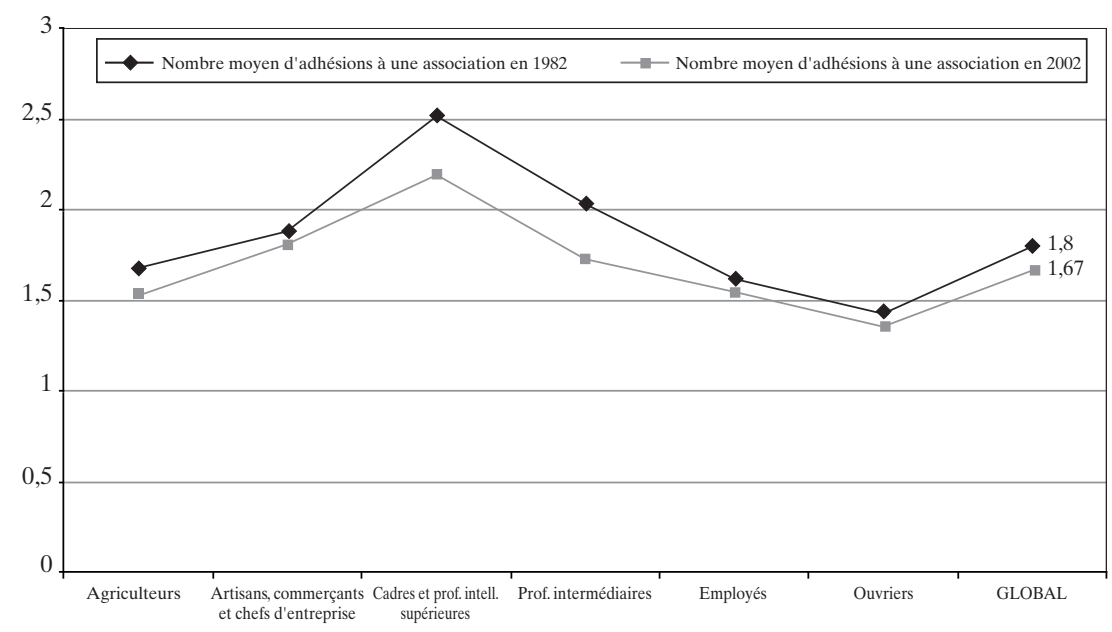

9. Nombre moyen d'adhésions par tranche d'âge parmi les adhérents d'au moins une association

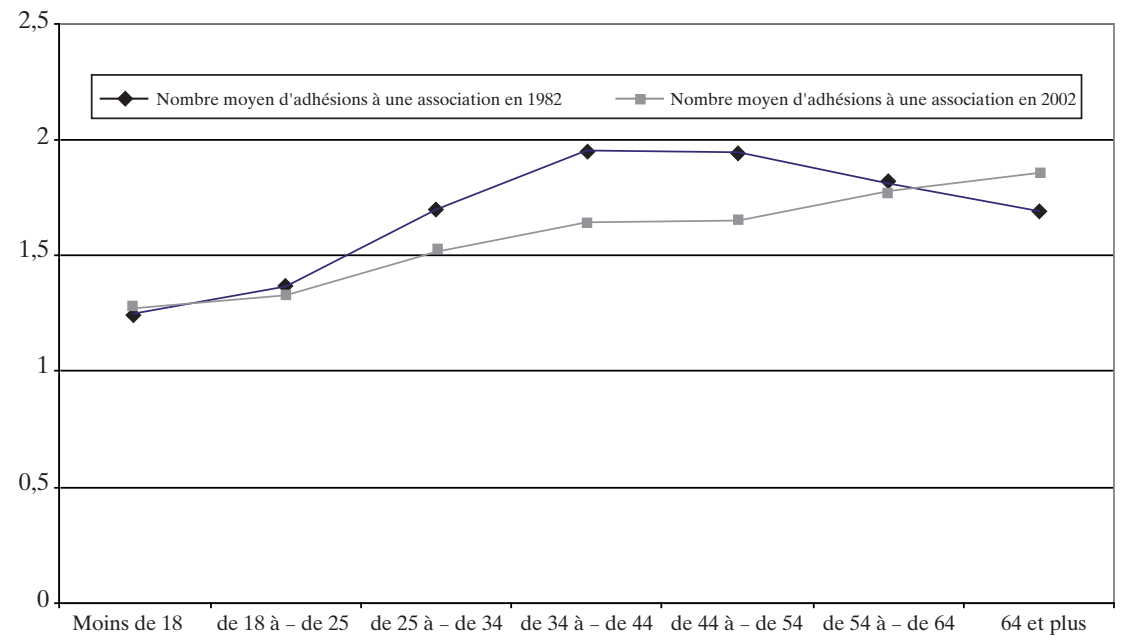

responsabilité est suffisamment importante pour être significative en dépit de l'erreur d'estimation liée à la taille des échantillons. À l'inverse, le taux des simples adhérents est en forte baisse, comme l'attestent les deux tableaux ci-dessous. 
10. Évolution des degrés de participation entre 1982 et 2002

En 1982

\begin{tabular}{|c|c|c|}
\hline $\begin{array}{c}\text { Type d'adhérents } \\
\text { (selon l'adhésion la plus élevée) }\end{array}$ & Effectif & Taux d'adhérents \\
\hline Simple adhérent (DP 1) & 2262 & $36,8 \%$ \\
\hline Participation active (DP 2) & 2747 & $44,7 \%$ \\
\hline Avec responsabilité (DP 3) & 1133 & $18,4 \%$ \\
\hline Total & 6142 & $100 \%$ \\
\hline
\end{tabular}

En 2002

\begin{tabular}{|c|c|c|}
\hline $\begin{array}{c}\text { Type d'adhérents } \\
\text { (selon l'adhésion la plus élevée) }\end{array}$ & Effectif & Taux d'adhérents \\
\hline Simple adhérent (DP 1) & 489 & $18,6 \%$ \\
\hline Participation active (DP 2) & 1577 & $60,1 \%$ \\
\hline Avec responsabilité (DP 3) & 559 & $21,3 \%$ \\
\hline Total & 2625 & $100 \%$ \\
\hline
\end{tabular}

Il convient de noter aussi que la tendance diffère selon le type d'association. Si le niveau de participation s'élève effectivement entre 1982 et 2002 tous secteurs associatifs confondus, on observe que c'est dans les secteurs des parents d'élèves, des groupements professionnels et des personnes âgées que le DP 2 augmente le plus fortement et que c'est dans les secteurs de la culture, de la musique et du sport que le DP 3 augmente le plus fortement. Il est ainsi notable de remarquer que c'est dans les secteurs apparemment les plus ludiques (sport, culture et musique) que le degré de responsabilité s'élève le plus.

\section{Conclusion}

Après avoir vérifié l'effet déterminant et immuable des variables sociodémographiques sur la probabilité d'adhérer à une association, l'analyse statistique fondée sur des données ponctuelles répétées entre 1982 et 2002 met en lumière quelques tendances significatives des transformations contemporaines de l'acteur associatif: les «baby-boomers»y occupent désormais une position dominante au détriment des plus jeunes. Il est d'ailleurs probable que l'allongement de la durée des études conjugué à une dégradation des conditions d'entrée sur le marché du 
travail soient des facteurs qui rendent l'adhésion et l'engagement, dont la gratuité n'est qu'une apparence trompeuse, fortement «coûteux ». Par ailleurs, il semble bien que ceux qui adhèrent dans au moins une association en 2002 sont beaucoup plus nombreux qu'en 1982 à s'investir et à prendre des responsabilités. Ce processus de « professionnalisation » est souvent souligné dans de nombreux travaux : il se caractérise par un nombre croissant de salariés ${ }^{17}$, une rationalisation des conditions d'exercice de la pratique bénévole ${ }^{18}$, ou encore un lien de plus en plus attesté entre l'engagement associatif et l'activité professionnelle (lien par les compétences transférables ou lien d'intérêt ${ }^{19}$ ). Notons que cette « professionnalisation » est aussi soulignée à travers de nombreuses publications extra-académiques (études, ouvrages de management ${ }^{20}$ ). Pour nous, cette « professionnalisation » se caractérise par une élévation significative du degré de participation des adhérents et une part croissante des diplômés de l'enseignement supérieur (alors que les moins diplômés apparaissent littéralement évincés de la sphère de l'engagement). On peut résumer l'évolution de l'engagement associatif comme ceci : moins mais mieux, moins de cumul d'adhésions mais plus de responsabilités. Bref, ces premiers résultats, qui en appellent d'autres à venir, confortent pour l'instant l'idée selon laquelle la figure contemporaine du bénévole (et notamment parmi ceux que l'on peut qualifier de « dirigeants » au sens où ils exercent des fonctions d'administration et de direction du groupement) se serait profondément transformée : le temps semble loin où la seule «bonne volonté » pouvait être invoquée comme une justification de l'engagement. L'expérience acquise par la formation initiale et au cours de la vie professionnelle apparaît désormais une ressource indispensable à toute implication dans la société civile. De même, le cumul d'adhésions est plus faible aujourd'hui qu'en 1982. Comme si l'engagement associatif nécessitait désormais une véritable spécialisation qui ne laisserait plus le temps à une dispersion des adhé-

17. Hély M., «Les différentes formes d'entreprises associatives », Sociologies Pratiques, $\mathrm{n}^{\circ} 9,2004$, p. 27-51.

18. Simonet M., «Penser le bénévolat comme travail pour repenser la sociologie du travail », Revue de l'IRES, no 44, 1, p. 141-155.

19. Bernardeau D., Sociologie des fédérations sportives. La professionnalisation des dirigeants bénévoles, Paris, L'Harmattan, coll. «Logiques sociales », 2004.

20. Baculard O., "Bénévolat de compétences, une nouvelle forme de mécénat », Volonteer, 2006 ; Belaroussi D. et Lasserre S., Manager les bénévoles, Dalloz-Juris Associations, Paris, 2005 ; La Tribune Fonda, $\mathrm{n}^{\circ}$ 174, août 2005, qui restitue les résultats d'une étude sur la Validation des Acquis de l'Expérience (VAE) des bénévoles. 
sions. Aurions-nous affaire à de véritables «professionnels » du bénévolat? La gratuité et la générosité ne sont plus en effet des critères suffisants pour qualifier la pratique bénévole. Celle-ci s'affichant de plus en plus comme un «travail » faisant appel à des compétences spécifiques. Le monde associatif apparaît ainsi comme une réalité ambivalente, oscillant en permanence « entre le travail et l'engagement » pour reprendre le titre d'une contribution récente de Maud Simonet ${ }^{21}$.

\section{Sur la méthodologie des enquêtes et la construction des échantillons}

En 1982, le fichier contient 6142 adhérents cumulant 10346 adhésions. En 2002, le fichier contient 2625 adhérents cumulant 3871 adhésions. L'enquête « contacts » a été menée de mai 1982 à mai 1983 conjointement par l'INSEE et l'INED auprès d'un échantillon de 5900 ménages (16 400 individus au total). Pour chacun des ménages interrogés, la personne enquêtée devait être âgée d'au moins 18 ans. L'échantillon se compose de 8104 logements dont 7027 à partir du recensement de la population de 1975 et 1077 dans la liste des logements neufs construits depuis 1975. On dénombre 6142 personnes qui déclarent « être membre d'une association ». L'enquête permanente sur les conditions de vie des ménages (EPCV) comporte depuis 1996 une série de questions sur le thème de la participation associative dont certaines sont similaires à l'enquête " contacts ». Ce qui autorise une comparaison entre ces deux périodes. L'échantillon est composé de 8000 ménages dans lesquels une personne âgée de 15 ans ou plus (et tirée au sort) a été interrogée. 5799 ménages ont répondu à la fois à la partie fixe de l'enquête et à la partie variable de 2002 qui concernait la participation associative. On dénombre 2625 personnes qui déclarent être membre d'au moins une association.

dbernardeaumoreau@free.fr mhely@u-paris10.fr

21. Simonet M., «Le monde associatif : entre travail et engagement », in Alter N. (dir.), Sociologie du monde du travail, Paris, PUF, 2006, p. 191-207. 